\title{
In-vitro prostate cancer biomarker detection by directed conjugation of anti-PSCA antibody to super paramagnetic iron oxide nanoparticles $s$
}

\author{
Mahboube Shahrabi Farahani ${ }^{1}$, Monireh Mohsenzadegan², Jaleh Taeb ${ }^{3}$, Mohammad M Farajollahi*1,3
}

Received: 21 May 2018

Published: 12 March 2019

\section{Abstract}

Background: The main property of a successful conjugation of antibodies to nanoparticles is keeping the potency of antibody for binding the antigen, and an oriented conjugation can do that. Under such ground, this study was carried out to explore the efficiency of two conjugation methods in binding iron nanoparticles to an antibody produced against PSCA (prostate stem cell antigen) using in vitro labeling of PC3 cells.

Methods: In this experimental study, we conjugated dextran-superparamagnetic iron oxide nanoparticles (dexSPIONs) to anti-PSCA antibody by two different methods, including targeting carbohydrate moieties in FC domain and the free amine group of amino acid side chains. Ultimately, Iron staining was done by anti-PSCA antibody-dexSPIONs in PC3 cells to detect antibody binding to the cells.

Results: A strong blue dye was induced by iron staining in conjugated dexSPIONs on the membrane of PC 3 cells by the former method than the second one. Moreover, cells treated with $20 \mathrm{~nm}$ diameters of dexSPIONs showed higher resolution of blue color than those treated with $100 \mathrm{~nm}$ nanoparticles.

Conclusion: This oriented conjugation method promoted the efficiency of targeting tumor antigens, and the presence of iron particles might enhance MRI image intensity in vivo by targeting PSCA-overexpressing cells in future studies.

Keywords: Anti-PSCA antibody, Conjugation, DexSPIONs, PC3 cells

Conflicts of Interest: None declared

Funding: Iran University of Medical Sciences

\section{*This work has been published under CC BY-NC-SA 1.0 license. \\ Copyright $\odot$ Iran University of Medical Sciences}

Cite this article as: Shahrabi Farahani M, Mohsenzadegan M, Taeb J, Farajollahi MM. In-vitro prostate cancer biomarker detection by directed conjugation of anti-PSCA antibody to super paramagnetic iron oxide nanoparticles. Med J Islam Repub Iran. 2019 (12 March);33:16. https://doi.org/10.47176/mjiri.33.16

\section{Introduction}

Cancer theranostics has been considerably improved with the advent and rapid progress of nanotechnology (1). Developing nanoscience-related strategies have provided more efficient and noninvasive approaches without seriously damaging the healthy tissue in comparison with traditional cancer treatment methods such as chemotherapy (1).

Corresponding author:Dr Mohammad M Farajollahi, fam-biotec@iums.ac.ir

1. Department of Medical Biotechnology, Faculty of Allied Medical Sciences, Iran University of Medical Sciences, Tehran, Iran

2. Department of Medical Laboratory Science, Faculty of Allied Medical Sciences, Iran University of Medical Sciences, Tehran, Iran

3. Cellular \& Molecular Research Center, Iran University of Medical Sciences, Tehran, Iran
Among numerous nanomaterials studied in the cancer research field, superparamagnetic iron oxide nanoparticles (SPIONs) have been considered one of the most attractive candidates. In magnetic resonance imaging (MRI), the SPIONs can be applied as a contrasting agent for diagnosis and treatment, due to their super-paramagnetism fea-

$\uparrow$ What is "already known" in this topic:

There are a few studies that have assessed the oriented conjugation methods (Covalent binding via carbohydrate moieties on the FC region of antibody). The conjugation of anti-hCG antibody to paramagnetic nanoparticles is one of them which was tested successfully.

\section{$\rightarrow$ What this article adds:}

In this study the oriented conjugation method was done through carbohydrate moieties of the FC domains for antiPSCA antibody and dextran-superparamagnetic iron oxide nanoparticles for the first time in the world. In comparison with non-oriented conjugation, this method efficiently enhances the targeting of prostate tumor antigens in vitro. 
ture $(2,3)$. These nanoparticles have interestingly low toxicity and are biodegradable. Furthermore, the small size of SPIONs facilitates the endocytosis and penetration into tissues and enhances drug-loading, which all make them the ideal candidate for molecular targeting (1, 4-6).

In spite of the potential characteristics of SPIONs, using the unconjugated nanoparticles may lead to weak binding, which can accumulate on target cells. In other words, they supply detection with low sensitivity and specificity. Fortunately, SPIONs can be simply bound to different types of molecules such as antibody, peptide, and aptamer without compromising their affinity, targeting specific cancer biomarkers on the tumor cells (7). This plan would enhance the selective accumulation on target cells, which express high amounts of correlated biomarkers or receptors on their surfaces. Subsequently, the desired effect can be augmented without leaking to adjacent healthy tissues. Antibodies are one of the most used biomolecules to meet the aim owing to their innate high specificity and large variety. Lots of tumor-associated antigens have been found as ideal candidates that can be targeted by antibodies (8).

Prostate stem cell antigen (PSCA) is a cell membrane marker that has been discovered to be overexpressed specifically in prostate cancer; however, its expression is limited in normal tissues $(6,9)$. The presence of this antigen is strongly associated with Gleason score, and it is upregulated in metastatic castration-resistant prostate cancer (mCRPC) lacking medication options (7, 10). Consequently, PSCA can be considered a significant prognostic marker in prostate cancer imaging $(1,11)$, and evaluation of its expression on biopsies or blood screen can provide valuable information for clinicians regarding the aggressiveness and/or susceptibility to the metastatic behavior of established tumors $(5,12,13)$. Consequently, the antibody against PSCA can be an appropriate option to be applied in actively targeting cancerous prostate cells by means of conjugation to nanoparticles.

Conjugation of antibody to nanoparticles such as SPIONs is a classical approach to target the cancer biomarkers on the cell surfaces through specific antibodyantigen interaction. Studies have shown that antibodyconjugated nanoparticles have been used promisingly for a variety of biotechnological and biomedical manipulations $(7,14)$. Although many advances have been achieved in the conjugation, the purposeful bioconjugation of nanoparticles, which sustains the biological function and antigen binding site of the antibody successfully in the application of the antibody-conjugated nanoparticles, is still a challenge (14). To have a successful targeting, the conjugation method for each nanoparticle and antibody is required to be optimized. Several oriented conjugation methods have been presented, one of which is covalent binding via carbohydrate moieties on the $\mathrm{FC}$ region of an antibody (15). In the current study, we conjugated dexSPIONs to the newly prepared anti-PSCA antibody against recombinant PSCA (rPSCA) protein by two different methods, including oxidizing carbohydrate moieties of FC regions and targeting the amine group of amino acid side chains of anti-PSCA antibody using sulfo-NHS. Iron staining was carried out to assess and compare the intensity of staining by anti-PSCA antibody-dexSPIONs by these methods in PC3 cells. Moreover, we compared the staining pattern of conjugated dexSPIONs with 20 and $100 \mathrm{~nm}$ in diameter by oxidizing carbohydrates of anti-PSCA antibody.

\section{Methods}

\section{Cell culture}

A cell line of human prostate cancer, PC3, was obtained from the Pasteur Institute of Iran. The cell line was seeded in a medium called Roswell Park Memorial Institute (RPMI) (GIBCO, Rockville, MD), associated with 10\% fetal bovine serum (FBS) (GIBCO) and $100 \mu \mathrm{g} / \mathrm{ml}$ streptomycin /penicillin (GIBCO). The cells were cultured in a $95 \%$ air humidified and $5 \% \mathrm{CO}_{2}$ incubator for 10 days at $37^{\circ} \mathrm{C}$.

\section{Preparation of rPSCA protein and anti-PSCA polyclonal} antibody

In the first phase of this experimental in vitro study, rPSCA protein and anti-PSCA polyclonal antibody were produced and purified for the next phase of conjugation assay. Expression vector for the human full PSCA (1-123 aa) $6 \times$-histidine (His) was constructed by subcloning the PSCA sequences into the pET28a vector between NdeI and XhoI enzyme restriction sites (GenScript Co., Ltd, USA, Inc). For affinity purification of rPSCA, the designed vector had a $\mathrm{C}$-terminal $6 \times$-His tag before the stop codon. Escherichia coli (E.coli) BL21 strain (DE3, Pasteur Institute, Iran) was applied for rPSCA expression. Transformation and expression in competent cells of $E$. coli BL21 were done as previously described (16). Finally, purification of the His-tagged PSCA protein was performed by a Ni-NTA column (Invitrogen, CA, USA) based on the manufacturer's instructions and tested for purity and immunoreactivity using sodium dodecyl sulfate polyacrylamide gel electrophoresis (SDS-PAGE) followed by staining with Coomassie blue (data not shown) and immunoblotting, respectively (17).

Two rabbits were bought from the Pasteur Institute of Iran and injected with the purified rPSCA as emulsion associated with complete Freund's adjuvant (first injection) and/or incomplete adjuvant (next injections). This vaccine was subcutaneously injected in several parts on the back of the rabbits. The first injection was performed with $300 \mu \mathrm{g}$ of rPSCA associated with Freund's complete adjuvant. In subsequent doses, a concentration of $150 \mu \mathrm{g}$ of rPSCA was injected at three different times with two weeks interval. Each injection was combined with equal volumes of complete adjuvant and/or incomplete adjuvant. The blood was collected from the central artery of the rabbits' ear before immunization and 10 days after each injection. ELISA was run to detect titrations of specific antibodies in the antisera based on a method previously determined by the corresponding author (18). 
sayed via ELISA. Briefly, flat wells of ELISA plates (Nunc, Rochester, NY, USA) were coated with $3 \mu \mathrm{g} / \mathrm{ml}$ of rPSCA in $100 \mathrm{mM}$ carbonate bicarbonate buffer ( $\mathrm{pH}$ 7.2) and put at $4^{\circ} \mathrm{C}$ for 24 hours (h), the wells were then blocked. One hundred $\mu 1$ of immunized rabbit serum was serially diluted, poured into the wells and put at $37^{\circ} \mathrm{C}$ for $1 \mathrm{~h}$. Goat anti-rabbit IgG as secondary antibody conjugated to horseradish peroxidase (HRP) (Santa Cruz Biotechnology, CA, USA) was then added in a 1:2000 dilution into the wells and incubated at $37^{\circ} \mathrm{C}$ for $1 \mathrm{~h}$, and the wells were washed afterward (19). Tetramethyl benzidine liquid substrate (TMB, Roche) as a chromogenic substance was added to the wells, and color progression was stopped by $0.2 \mathrm{M}$ sulfuric acid. The data were picked up at the optical density (OD) of $450 \mathrm{~nm}$ by an ELISA reader (model 680, Bio-Rad, CA, USA).

For purification of anti-PSCA antibody from anti-rabbit sera, the rPSCA was chemically bound to the beads of NHS-activated agarose (Pierce Chemicals Co., IL, USA) and purified according to the manufacturer's methods (20). The amount of the purified anti-PSCA antibodies was assayed by BCA kit (Thermo Fisher Scientific, IL, USA) using the BSA (Bovine Serum Albumin) standard curve.

\section{Protein extraction \& western blotting analysis}

Cultured PC 3 cells were trypsinized and dissolved in 1 M lysis buffer (2.4 gr urea, 5.1 gr thiourea, 4 gr CHAPS, 4 $\mathrm{ml}$ of Tris buffer in $10 \mathrm{ml}$ deionized water) and protease inhibitor cocktail (Sigma, MO, USA). Protein concentration in the supernatant was assayed by the BCA kit. Cell lysates and/or rPSCA protein $(50 \mu \mathrm{g})$ were blended and boiled by loading buffer for $5 \mathrm{~min}$ and loaded onto $7 \%$ SDS-PAGE plus $10 \%$ SDS and $\beta$-mercaptoethanol. The proteins were transferred onto the polyvinylidene difluoride (PVDF) membrane $\left(240 \mathrm{~mA}\right.$ for $45 \mathrm{~min}$ at $\left.4^{\circ} \mathrm{C}\right)$ in transfer buffer of a wet western blot apparatus; the membrane was then blocked for $1.5 \mathrm{~h}$ at RT (21). Afterward, $1: 1000$ dilution of anti-PSCA antibody $(0.8 \mathrm{mg} / \mathrm{ml})$ was added to the membrane in TBS, incubated overnight at $4^{\circ} \mathrm{C}$ and washed six times with TBS-T buffer. The goat anti-rabbit HRP-conjugated antibody (1:60000, Abcam, MA, USA) was then added for $1 \mathrm{~h}$ at RT. Finally, bands were exposed by enhanced chemiluminescence (ECL) (Pierce) and presented on an x-ray film (Roche).

\section{Immunocytochemistry}

The PC3 cells grew in 24 -well plates at $37^{\circ} \mathrm{C} / 5 \% \mathrm{CO}_{2}$ for $24 \mathrm{~h}$. The cells were then washed with $100 \mathrm{mM}$ PBS-T (pH 7.2), fixed in $4 \%$ paraformaldehyde (V/V) for $20 \mathrm{~min}$, and blocked with 5\% BSA in PBS-T for 45 min at RT. Subsequently, the cells were treated with anti-PSCA antibody as primary antibody diluted 1:1000 for $1 \mathrm{~h}$ at RT. The goat anti-rabbit IgG conjugated to FITC (Sigma) was added with a 1:160 dilution for $1 \mathrm{~h}$ at RT, and subsequently the nuclei of cells were stained with Hoechst solution (Thermo Fisher) and analyzed using an inverted fluorescence microscope (Olympus, Japan). The plates were washed with PBS-T (pH 7.2) between each step. PBS and equal concentration of $\operatorname{IgG}$ fraction from pre-immunized rabbit serum were applied as negative controls instead of the primary antibody (22).

\section{Oriented conjugation of purified polyclonal anti-PSCA} antibody to SPIONs

Preparation of Hydrazide-Activated SPIONs: SPIONs with a cross-linked dextran coating (dexSPIONs) containing-COOH group (nanomag $\mathbb{\circledR}-\mathrm{CLD}$-spio $100 \mathrm{~nm}$, Micromod Partikeltechnologie GmbH, Rostock, Germany) and $20 \mathrm{~nm}$ dexSPIONs, a gift from Nanochem Dept, Tehran University, were functionalized by adipic acid dihydrazide (Sigma Aldrich, USA). Briefly, $8 \mathrm{mg}$ of adipic acid dihydrazide was dissolved in $250 \mu \mathrm{l}$ of sodium phosphate $(0.1$ $\mathrm{M}$; pH 6.0), and then $2.5 \mathrm{mg}$ of each dexSPIONs were dissolved in the prepared adipic acid dihydrazide solution. Eight $\mathrm{mg}$ of water-soluble carbodiimide 1-Ethyl-3-(3dimethylaminopropyl) carbodiimide (EDC) (Thermo Fisher, USA) were added and blended to them to dissolve completely (Fig. 1). The reaction was incubated for $4 \mathrm{~h}$ at RT. Finally, to remove the excess reagent and reaction byproduct, the solution was dialyzed against PBS $(0.15 \mathrm{M}$; $\mathrm{pH} 7.2)$ overnight at $4^{\circ} \mathrm{C}(23)$.

Activation of antibodies with sodium periodate: An appropriate amount of anti-PSCA polyclonal antibody was dissolved in sodium phosphate $(0.01 \mathrm{M}$; pH 7.2) and 0.15 $\mathrm{M} \mathrm{NaCl}$. The carbohydrate moieties of anti-PSCA antibody $(1 \mathrm{mg} / \mathrm{ml})$ were oxidized by $20 \mu \mathrm{l}$ of sodium periodate solution $(0.1 \mathrm{M})$ (Fig. 1). The reaction was done in a dark room for $20 \mathrm{~min}$ and quenched by addition of sodium sulfite $\left(\mathrm{Na}_{2} \mathrm{SO}_{3}\right)$. Ultimately, the oxidized antibody was purified using gel filtration via a desalting resin (G-50). One $\mathrm{ml}$ fractions were collected, and the protein content of the fractions was measured using a NanoDrop spectrophotometry (Thermo Onec, USA) at $280 \mathrm{~nm}$ of light absorbance (23).

After oxidizing the antibody by sodium periodate, conjugation was done by addition of $1 \mathrm{ml}$ of desalted oxidized anti-PSCA antibody $(100 \mu \mathrm{g} / \mathrm{ml})$ and $500 \mu \mathrm{l}$ of Hydrazide-Activated SPIONs $(5 \mathrm{mg} / \mathrm{ml})$ mixed and put at RT for $2 \mathrm{~h}$, followed by blocking the unreacted aldehyde sites of the antibody through addition of $1 \mathrm{M}$ ethanolamine, $\mathrm{pH}$ 9.6. The compound was incubated at RT for $30 \mathrm{~min}$. The unconjugated anti-PSCA antibody was removed using the MACS magnetic column (Miltenyi Biotec, Germany) (24) to obtain the resulting conjugated anti-PSCA antibodyfunctionalized iron oxide nanoparticles (Fig. 1A).

Conjugation of anti-PSCA antibody via amine groups of amino acid: In another experiment, $1 \mathrm{mg} / \mathrm{ml}$ of dexSPIONs containing - $\mathrm{COOH}$ groups was activated by $0.05 \mathrm{M}$ of 2-(N-morpholino) ethanesulfonic acid (MES) and 0.5 $\mathrm{M} \mathrm{NaCl}(\mathrm{pH} 6.0)$. The activated nanoparticles were added to $2 \mathrm{mM}$ EDC and $5 \mathrm{mM}$ sulfo-NHS (Thermo Fisher) for $15 \mathrm{~min}$ at RT to constitute the sulfo-NHS esters. The activated nanoparticles were purified from the reaction by gel filtration using a desalting resin. To conjugate, the activated nanoparticles were added to the anti-PSCA antibody dissolved in sodium phosphate $(0.1 \mathrm{M} ; \mathrm{pH} 7.5)$ for $2 \mathrm{~h}$ at RT. The unconjugated anti-PSCA antibody was purified 

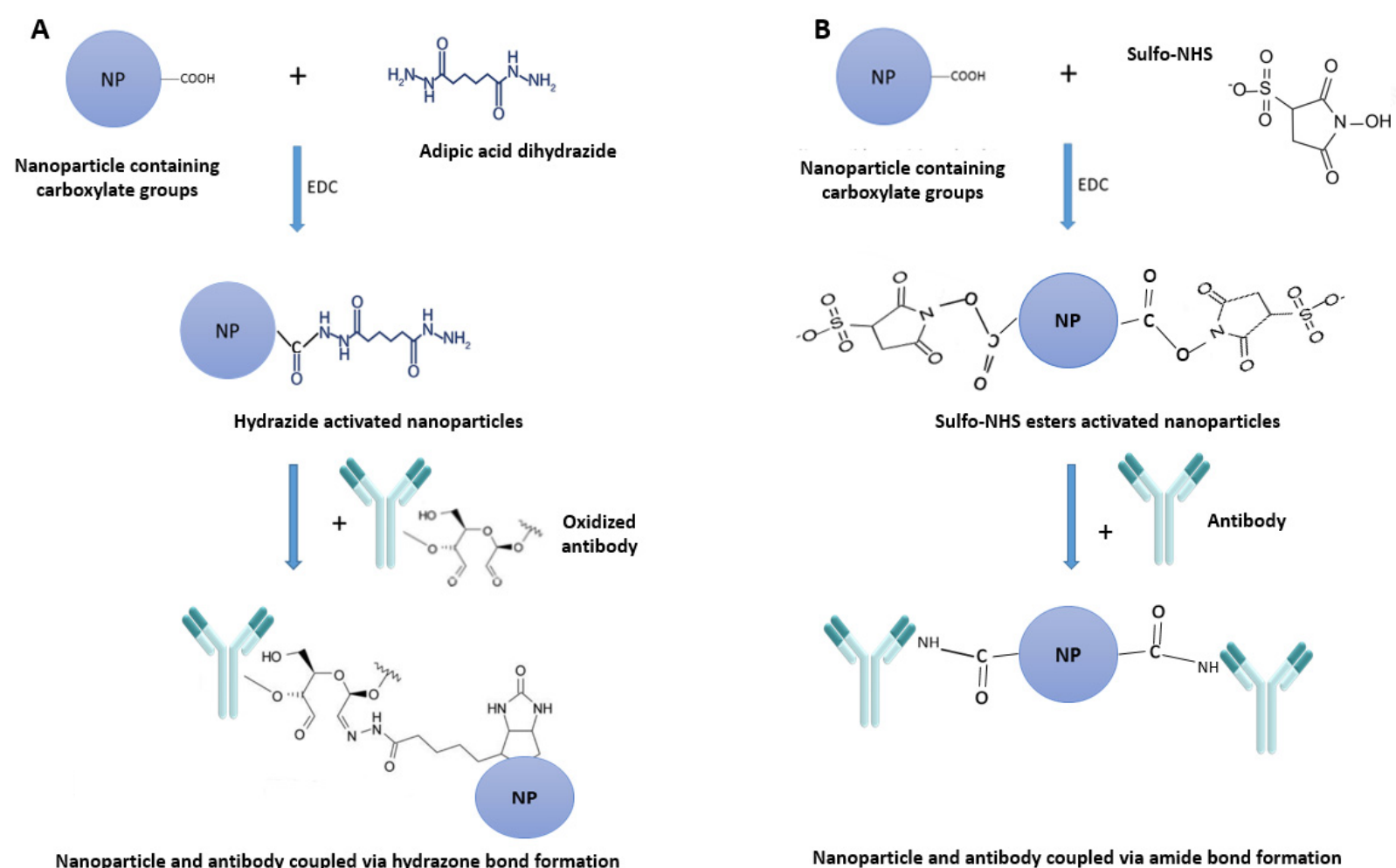

Fig. 1. Scheme of bioconjugation for anti-PSCA antibody-dexSPIONs. (A) DexSPIONs were functionalized by adipic acid dihydrazide via EDC. Carbohydrate positions of antibody were oxidized using sodium periodate (not shown) and linked toward activated dexSPIONs. (B) DexSPIONs were activated by MES (not shown), bound to EDC and sulfo-NHS and formed the sulfo-NHS esters. DexSPIONs containing sulfo-NHS esters were conjugated to anti-PSCA antibody through amine groups of antibody.

using the MACS magnetic column to get rid of un-reacted free antibodies (Fig. 1 B) (23).

Cell staining by Perl's Prussian blue: The PC3 cells were seeded in 12-well plates and incubated for $24 \mathrm{~h}$ at $37^{\circ} \mathrm{C} / 5 \% \mathrm{CO}_{2}$. They were washed with PBS-T, fixed for $20 \mathrm{~min}$ in $4 \%$ paraformaldehyde $(\mathrm{V} / \mathrm{V})$, and incubated with $5 \% \mathrm{BSA} / \mathrm{PBS}-\mathrm{T}$ for $45 \mathrm{~min}$ at RT. Subsequently, PC3 cells were incubated with anti-PSCA antibody conjugated dexSPIONs $(70 \mu \mathrm{g} / \mathrm{ml})$ and/or free dexSPIONs (as a control) for $2 \mathrm{~h}$ at RT. The dexSPIONs that mostly attached to the cell surface were stained using Prussian Blue Iron Stain Kit (Polysciences, USA), and to stain the nucleus; Nuclear Fast Red solution (Polysciences) was used. The plates were washed with PBS-T between each step. The PC3 cells were investigated under a light microscope (Olympus IX51; Olympus, Tokyo, Japan) at 10X and 40× magnifications. The AGS cell line (human epithelial stomach) was applied as a negative control cell $(25,26)$.

\section{Results}

\section{Evaluation of anti-rPSCA anti-serum}

It has been established that rPSCA protein is immunogenic because the production of the anti-rPSCA antibody is strongly induced in the rabbit. The anti-sera showed reactivity with rPSCA by ELISA; whereas, IgG fraction of the rabbit pre-immune serum did not have any reactivity. ELISA results showed that antibody titers were convincingly high at $>1 / 6400$ dilution (Fig. 2 A). The specific antibodies purified by affinity chromatography were tit- tered and determined at 1:12800 dilutions. The IgG fraction of pre-immunized rabbit at the same dilutions was applied as a negative control (Fig. 2 B).

\section{Immuno-reactivity of anti-PSCA antibody by western} blotting analysis

To evaluate the specificity of anti-sera, Western blot analysis was done on PC3 and rPSCA to assay the expression of PSCA by anti-PSCA antibody. The cell lysates and rPSCA were analyzed on a PVDF membrane, as shown in Fig. 2 C. Specific bands approximately 26 (Fig. 2 C1) and $15 \mathrm{kDa}$ (Fig. 2 C2) were detected by anti-PSCA antibody in PC3 cells and rPSCA, respectively. The rPSCA produced in prokaryotic system migrates at a lower molecular weight than the cell line lysates, perhaps reflecting the absence of the covalent glycosylphosphatidylinositol (GPI)-linkage. The PSCA expression was almost high and moderate in PC3 and E. coli BL21 cells, respectively (Fig. $2 \mathrm{C}$ ), while no band was found for non-immunized serum (data not shown). These results indicated that the produced anti-PSCA antibody could effectively recognize both human exogenous rPSCA and endogenous PSCA.

Immunocytochemical analysis of PC3 cells using antiPSCA antibody

We analyzed the staining pattern of PSCA in prostate cancer cells treated with anti-PSCA antibody by immunocytochemistry. Immunocytochemical analysis of PC3 cell 

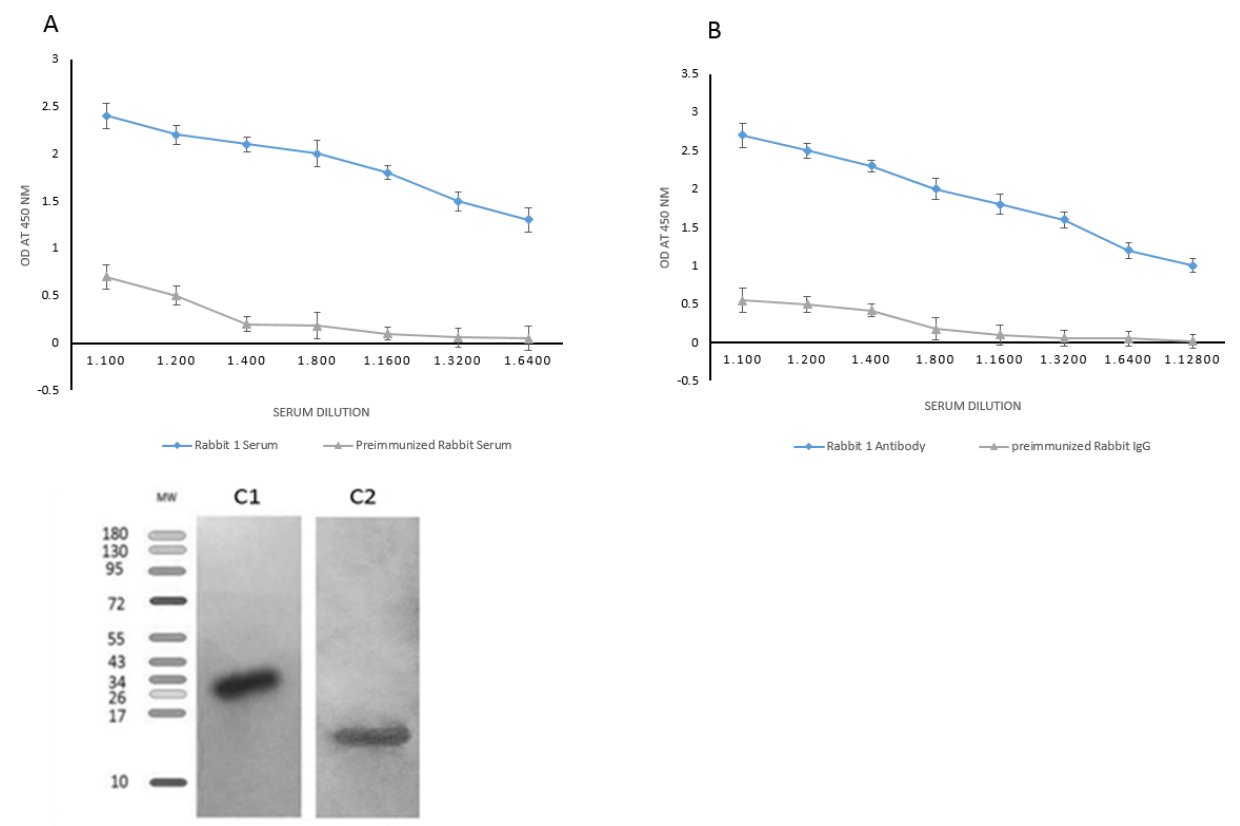

Fig. 2. Titration of anti-PSCA antisera by indirect ELISA (A) and the purified antibody against rPSCA (B). Western blot analysis of cell lysates (lane $\mathrm{C} 1$ ) and rPSCA (lane C2) detected using affinity purified anti-PSCA antibody (details have been described in the text).

A; FITC staining

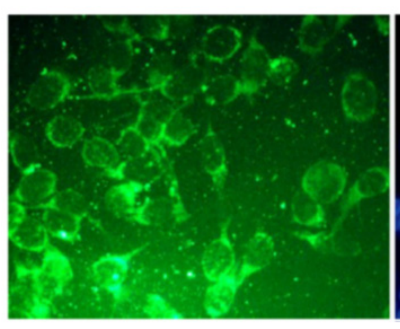

B; Hoechst staining (control)

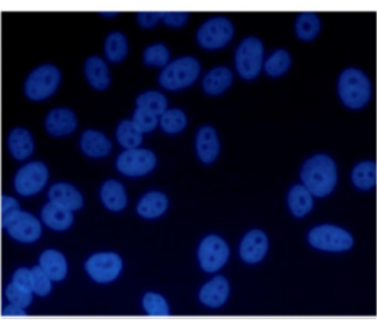

D; control c; merged imaged

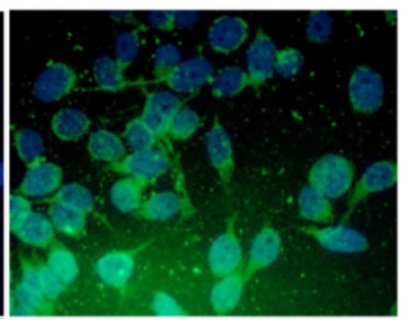

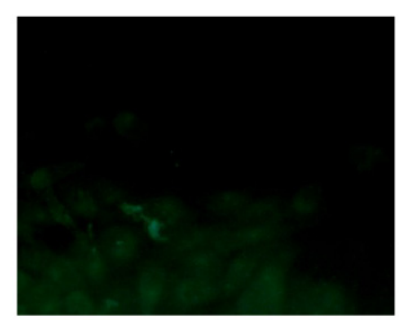

Fig. 3. Images of PC3 cell lines taken by immunofluorescence microscopy when treated with anti-PSCA antibody. A and C; PC3 cell lines incubated with anti-PSCA antibody, B and D; PC3 cells not incubated with anti-PSCA antibody (details have been described in the text).

line by anti-PSCA antibody showed green fluorescence signals mostly on the cell surface stained with only FITC and merged images stained with FITC and Hoechst dyes. When these cells were treated with pre-immunized rabbit serum at an equal concentration with anti-PSCA antibody, ghost cells with weak fluorescence signal were observed (Fig. 3).
Perl's Prussian blue staining of PC3 using anti-PSCA antibody-dexSPIONs

To characterize the specific binding of anti-PSCA antibody-dexSPIONs and to evaluate the successful conjugation toward PSCA-expressing tumor cells, iron staining was performed on PC3 cells using Perl's Prussian blue staining. The pattern (iron and nuclear fast red staining) was established to differentiate and detect the iron- 


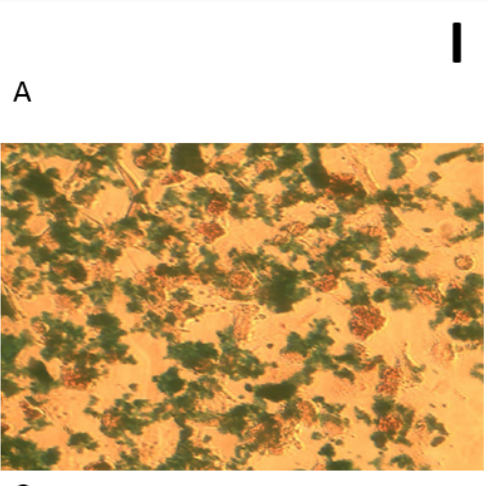

B

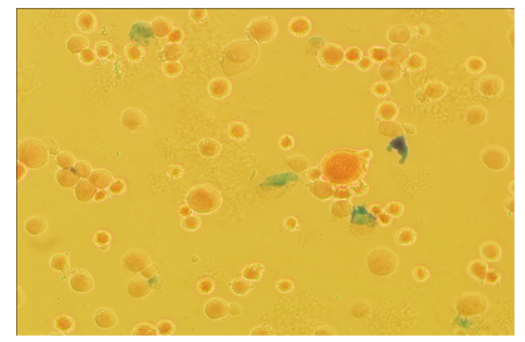

D

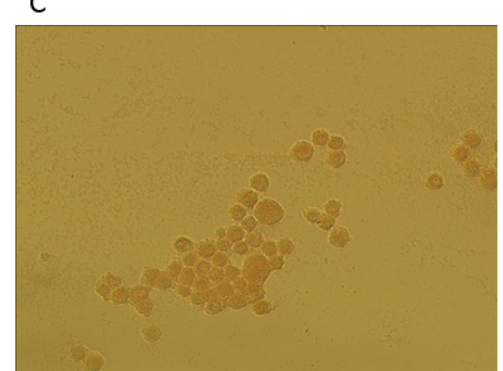

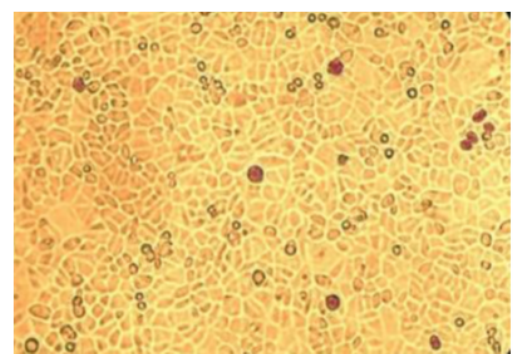

II

E

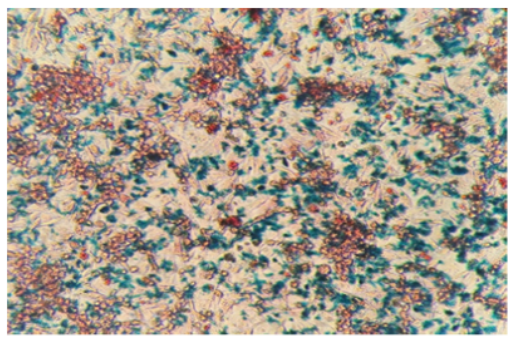

$\mathrm{F}$

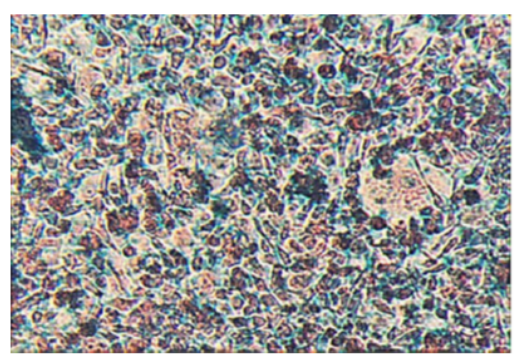

Fig. 4. I, PC3 cells stained with iron, after treatment with anti-PSCA antibody conjugated to dexSPIONs. A: Oriented conjugation of dexSPIONs to anti-PSCA antibody through carbohydrate moieties of antibody FC by adipic acid dihydrazide. B: Conjugation of anti-PSCA antibody via amine groups of amino acids by sulfo-NHS. C: PC3 cells were treated with non-conjugated dexSPIONs (negative control). D: AGS cells (negative control) treated with anti-PSCA antibody-dexSPIONs (details have been described in the text). II, Comparison of iron staining pattern between dexSPIONs with $20 \mathrm{~nm}$ and $100 \mathrm{~nm}$ diameters. E: dexSPIONs with $20 \mathrm{~nm}$ diameters conjugated to anti-PSCA antibody, F: dexSPIONs with 100 $\mathrm{nm}$ diameters conjugated to anti-PSCA antibody.

containing substances. In this study, targeted conjugation via oxidizing carbohydrates of antibody FC by sodium periodate and treatment of the PC3 cells with conjugated nanoparticles were performed. A strong blue dye appeared by iron staining and showed a high accumulation of conjugated nanoparticles on the membrane of PC3 cells by oriented conjugation via oxidizing carbohydrates of antiPSCA antibody in comparison with cells that were not treated with anti-PSCA antibody-dexSPIONs (Fig. 4 A, C). Cells treated with non-conjugated dexSPIONs (Fig. 4 C) and/or AGS cells (negative control; Fig. 4 D) treated with anti-PSCA antibody-dexSPIONs showed no blue stain, but their nuclei were stained. The results indicated that dex-SPIONs conjugated to anti-PSCA antibody via carbohydrate moieties of antibody FC strongly bound to PSCA in PC3 cells. However, when the amine groups of anti-PSCA antibody were targeted and conjugated to the activated dexSPIONs through sulfo-NHS, very weak signal in cells was observed in comparison with conjugation done by oxidizing carbohydrates of antibody (Fig. 4 A, B). The results indicated that targeted amine groups of antibody might affect the $F\left(a^{\prime}\right)_{2}$ amino acids responsible for antigen binding.

Moreover, we compared the staining pattern of conjugated dexSPIONs with 20 and $100 \mathrm{~nm}$ diameters by oxidizing the carbohydrates of anti-PSCA antibody. PC3 cells treated with dexSPIONs at $20 \mathrm{~nm}$ diameter showed higher resolutions of blue color absorbance, and staining pattern was more specific than those treated with $100 \mathrm{~nm}$ nanoparticles (Fig. 4 E, F). These findings showed that the small size of SPIONs could enhance permeability as well as retention effect and might lead to accumulation of the particles in target tumor cells.

\section{Discussion}

Nanoparticles have attracted intensive research efforts due to their unique properties and low side effects in patients with cancer therapy, and are also used for the treatment of other diseases. Among various types of nanoparticles, SPIONs have attracted great attention for targeting tumor antigens in cancer therapy and imaging applications (24, 27, 28). Furthermore, targeting cell-surface antigens is critical for developing the diagnostic and therapeutic algorithm for the management of prostate cancer. Among all prostatic cancer cell-surface antigens, we selected PSCA as a favorite target for in vitro imaging of prostate cancer cells for its overexpression on the prostate cancerous cell membrane (29). Moreover, PSCA is strongly expressed in both androgen-sensitive and in-sensitive prostate tumors as well as prostate cancer patients with high Gleason grades (Gleason grades: 4 and 5). In the present study, we produced recombinant full-length PSCA in the competent cells of $E$. coli BL21 followed by production of rabbit antibody against the protein. The analysis of ELISA results showed that rPSCA was immunogenic in rabbit such that the antibody produced against the rPSCA was at high titers. Our Western blot analysis represented a specific band at $26 \mathrm{kDa}$ when the antibody was used, indicating that the rabbit antibody produced by rPSCA can specifically react to the PSCA protein found in prostate cancer cells.

Herein, we conjugated anti-PSCA antibody to a dexSPIONs, as illustrated in Fig. 1. To activate dexSPIONs, we used two different chemical agents, including adipic 
acid dihydrazide and sulfo-NHS to alter the surface of the dextran-coated SPIONs. This process produced the activated dexSPIONs with hydrazide and/or sulfo-NHS ester groups for the subsequent antibody conjugation step. With two different methods, carbohydrate moieties in the oligosaccharide groups and amines of amino acid side chains of anti-PSCA antibody were targeted and then conjugated to the hydrazide and/or sulfo-NHS ester groups on the surface of the dexSPIONs to provide the antibodyfunctionalized dexSPIONs (anti-PSCA antibodydexSPIONs). Although there are a wide variety of oxidative reactions for aldehyde group formation of antibody carbohydrates, sodium periodate was the more favorable option due to lack of adverse reactions, forming aldehyde groups on the carbohydrate moieties of antibodies without changing the solubility of the antibody (14). When nanoparticles were conjugated through carbohydrate moieties of the FC domain, stronger signals were observed in comparison with a reaction in which it conjugated through amine side chains of the antibody. Gao et al. at 2012 targeted free sulfhydryl groups on $\mathrm{scAb}$ by using maleimidepolyethylene glycol-COOH linker to conjugate the SPIONs to single chain antibody against PSCA, (12). Given that the sulfhydryl groups may form in Fab area of antibody due to the presence of the cysteine group, this method may be involved in blocking the binding site of the antibody to the antigen. Shamsipour et al. conjugated antiher2/neu monoclonal antibody (Herceptin) to SPIO nanoparticles via amine groups of amino acid antibody using EDC method (30). The limitations of nanoparticle conjugation to an amine group of the antibody are due to the residues of the amino acids (such as lysine) in an antibody that accidentally bind to nanoparticle linkers, and the antigen-binding sites of antibody ( $\mathrm{F}(\mathrm{ab}) \mathrm{b}$ ) would not be presented towards tumor cell antigens. These specs may result in lower binding capacity and therefore affect the therapeutic and/or diagnostic application of the bioconjugation. Our results indicated that directed conjugation through carbohydrate moieties of the $\mathrm{CH} 2$ domains of antibodies could result in highly efficient detection of the prostate cancer cells by PSCA protein. Consequently, the method presented in this study can be successfully applied to the antibody modification reaction beyond the antigenbinding sites for the diagnosis of prostate cancer cells.

Furthermore, by decreasing the size of iron oxide particles into nanometer ranges, iron ions perform a major role on the surface of nanoparticles to do more efficient bioconjugation (31). Consequently, to increase the imaging sharpness and tumor detection accuracy with nanoparticles, it is essential to note the physical features of nanoparticles, including size and surface/volume ratio. It has been revealed that these properties of nanoparticles play a pivotal role in effective transport into tumor microcirculation (32). In general, renal clearance can clear the nanoparticles that are smaller than 5-10 nm from circulation, and by increasing the size of nanoparticles, they primary accumulate in the spleen and liver (33). To explore the efficacy of SPIONs with different sizes in imaging of prostate cancer cells, we compared the staining patterns of two different dexSPIONs with 20 and $100 \mathrm{~nm}$ in diameter by oxidizing the carbohydrate moieties of the antibody. Our findings showed that SPIONs with $20 \mathrm{~nm}$ diameter obtain a higher resolution in comparison with $100 \mathrm{~nm}$ diameter nanoparticles. The results indicated that the small size of SPIONs is also determinative for the retention effect on the tumor cells and increased permeability, which in turn leads to concentration of the nanoparticles on target cells. Therefore, controlling the nanoparticle size toward the favorable range can be effective in delivery vehicles that deal with tumor cells.

\section{Conclusion}

In the present study, we targeted PSCA antigen on the PC 3 cells by preparing anti-PSCA antibody conjugated to nanoparticles. The results showed strongly stained cells expressing PSCA by orientated conjugation of the antibody via carbohydrate groups of antibody FC region, suggesting a possible new approach for better cancer imaging and targeting strategies. Moreover, the findings showed that considering SPION size within the desirable range can result in better staining of tumor cells and may affect the penetration capacity of nanoparticles in tumor cells.

\section{Funding}

This study was conducted as a research project and supported by a grant from Iran University of Medical Sciences [Grant \#93-25077].

\section{Conflict of Interests}

The authors declare that they have no competing interests.

\section{References}

1. Kievit FM, Zhang M. Surface engineering of iron oxide nanoparticles for targeted cancer therapy. Acc. Chem. Res. 2011;44(10):853-62.

2. Chen HL, Hsu FT, Kao YJ, Liu HS, Huang WZ, Lu CF, et al. Identification of epidermal growth factor receptor-positive glioblastoma using lipid-encapsulated targeted superparamagnetic iron oxide nanoparticles in vitro. J Nanobiotechnology. 2017;15(1):86.

3. Moradi Khaniabadi P, Shahbazi-Gahrouei D, Jaafar MS, Majid A, Moradi Khaniabadi B, Shahbazi-Gahrouei S. Magnetic Iron Oxide Nanoparticles as T2 MR Imaging Contrast Agent for Detection of Breast Cancer (MCF-7) Cell. AJMB. 2017;9(4):181-8.

4. Zhu Y, Sun Y, Chen Y, Liu W, Jiang J, Guan W, et al. In vivo molecular MRI imaging of prostate cancer by targeting PSMA with polypeptide-labeled superparamagnetic iron oxide nanoparticles. IJMS. 2015;16(5):9573-87.

5. Ren J, Wang F, Wei G, Yang Y, Liu Y, Wei M, et al. MRl of prostate cancer antigen expression for diagnosis and lmmunotherapy. PloS one. 2012;7(6): e38350.

6. Mu K, Zhang S, Ai T, Jiang J, Yao Y, Jiang L, et al. Monoclonal Antibody-Conjugated Superparamagnetic Iron Oxide Nanoparticles for Imaging of Epidermal Growth Factor Receptor-Targeted Cells and Gliomas. MOL IMAGING. 2015;14(5):7290.2015. 00002.

7. Kumar S, Aaron J, Sokolov K. Directional conjugation of antibodies to nanoparticles for synthesis of multiplexed optical contrast agents with both delivery and targeting moieties. Nat. Protoc. 2008;3(2):314-20.

8. Dostalova S, Cerna T, Hynek D, Koudelkova Z, Vaculovic T, Kopel P, et al. Site-Directed Conjugation of Antibodies to Apoferritin Nanocarrier for Targeted Drug Delivery to Prostate Cancer Cells. ACS Appl. Mater. Interfaces. 2016;8(23):14430-41.

9. Saeki N, Gu J, Yoshida T, Wu X. Prostate stem cell antigen: a Jekyll and Hyde molecule? Clin. Cancer Res. 2010;16(14):3533-8.

10. Prieto-Simón B, Saint C, Voelcker NH. Electrochemical biosensors featuring oriented antibody immobilization via electrografted and selfassembled hydrazide chemistry. Anal. Chem. 2014;86(3):1422-9. 
11. Yang X, Guo Z, Liu Y, Si T, Yu H, Li B, et al. Prostate stem cell antigen and cancer risk, mechanisms and therapeutic implications. EXPERT REV ANTICANC 2014;14(1):31-7.

12. Gao X, Luo Y, Wang Y, Pang J, Liao C, Lu H, et al. Prostate stem cell antigen-targeted nanoparticles with dual functional properties: in vivo imaging and cancer chemotherapy. Int $\mathrm{J}$ Nanomedicine. 2012;7:4037.

13. Ling Y, Wei K, Luo Y, Gao X, Zhong S. Dual docetaxel/superparamagnetic iron oxide loaded nanoparticles for both targeting magnetic resonance imaging and cancer therapy. Biomaterials. 2011;32(29):7139-50.

14. Montenegro JM, Grazu V, Sukhanova A, Agarwal S, de la Fuente JM, Nabiev I, et al. Controlled antibody/(bio-) conjugation of inorganic nanoparticles for targeted delivery. Adv Drug Deliv Rev. 2013;65(5):677-88.

15. Montenegro J-M, Grazu V, Sukhanova A, Agarwal S, Jesus M, Nabiev I, et al. Controlled antibody/(bio-) conjugation of inorganic nanoparticles for targeted delivery. Adv Drug Deliv Rev. 2013;65(5):677-88.

16. Rosano GL, Ceccarelli EA. Recombinant protein expression in Escherichia coli: advances and challenges. Front Microbiol. 2014:7.

17. Hossain MB, Oshima T, Hirose S, Wang J, Tokumoto T. Expression and Purification of Human Membrane Progestin Receptor alpha (mPRalpha). PLoS One. 2015;10(9):e0138739.

18. Farajollahi MM, Cook DB, Hamzehlou S, Self $\mathrm{CH}$. Reduction of non-specific binding in immunoassays requiring long incubations. Scand J Clin Lab Invest. 2012;72(7):531-9.

19. Lin AV. Indirect ELISA. Methods Mol Biol (Clifton, NJ). 2015;1318:51-9.

20. Faramarzi R, Dolatabadi S. Expression and purification of recombinant HTLV-I/-II linear epitopes antigen and its application for screening of suspected patients. IJM. 2017;9(1):43-9.

21. Hnasko TS, Hnasko RM. The Western Blot. Methods Mol Biol (Clifton, NJ). 2015;1318:87-96.

22. Mohsenzadegan M, Tajik N, Madjd Z, Shekarabi M, Farajollahi MM. Study of NGEP expression in androgen sensitive prostate cancer cells: A potential target for immunotherapy. Med J Islam Repub Iran. 2015;29:159.

23. Hermanson GT. Bioconjugate Techniques: Elsevier Science; 2010.

24. Xu H, Aguilar ZP, Yang L, Kuang M, Duan H, Xiong Y, et al. Antibody conjugated magnetic iron oxide nanoparticles for cancer cell separation in fresh whole blood. Biomaterials. 2011;32(36):9758-65.

25. Chittamsetty H, Sekhar MS, Ahmed SA, Suri C, Palla S, Venkatesh $\mathrm{SM}$, et al. A Non-Invasive Technique which Demonstrates the Iron in the Buccal Mucosa of Sickle Cell Anaemia and Thalassaemia Patients who Undergo Repeated Blood Transfusions. JCDR. 2013;7(6):121922 .

26. Gajaria PK, Maheshwari UM, Borkar DB, Dhar R, Pancholi V. Buccal Mucosa Exfoliative Cell Prussian Blue Stain Co-Relates with Iron Overload in beta-Thalassemia Major Patients. Indian J Hematol Blood Transfus. 2017;33(4):559-64

27. Yeh CY, Hsiao JK, Wang YP, Lan CH, Wu HC. Peptide-conjugated nanoparticles for targeted imaging and therapy of prostate cancer. Biomaterials. 2016;99:1-15.

28. Martinez-Gonzalez R, Estelrich J, Busquets MA. Liposomes Loaded with Hydrophobic Iron Oxide Nanoparticles: Suitable T(2) Contrast Agents for MRI. Int J Mol Sci. 2016;17(8).

29. Ren J, Zhang Z, Wang F, Yang Y, Liu Y, Wei G, et al. MRI of prostate stem cell antigen expression in prostate tumors. Nanomedicine. 2012;7(5):691-703.

30. Shamsipour F, Zarnani A-H, Ghods R, Chamankhah M, Forouzesh F, Vafaei S, et al. Conjugation of monoclonal antibodies to super paramagnetic iron oxide nanoparticles for detection of her $2 /$ neu antigen on breast cancer cell lines. AJMB. 2009;1(1):27-31.

31. Wahajuddin SA. Superparamagnetic iron oxide nanoparticles: magnetic nanoplatforms as drug carriers. Int $\mathrm{J}$ Nanomedicine. 2012;7:3445.

32. Lee SY, Ferrari M, Decuzzi P. Shaping nano-/micro-particles for enhanced vascular interaction in laminar flows. Nanotechnology. 2009;20(49):495101.

33. Toy R, Bauer L, Hoimes C, Ghaghada KB, Karathanasis E. Targeted nanotechnology for cancer imaging. Adv. Drug Deliv. Rev. 2014;76:79-97. 